\title{
Generating a 3D Simulation of a Car Accident from a Written Description in Natural Language: the CarSim System
}

\author{
Sylvain DUPUY, Arjan EGGES, Vincent LEGENDRE, and Pierre NUGUES \\ GREYC laboratory - ISMRA \\ 6, bd du Maréchal Juin \\ F-14050 Caen, France \\ Email: \{dupuy,vlegendr\}@ensicaen.ismra.fr \\ pnugues@greyc.ismra.fr \\ egges@cs.utwente.nl
}

\begin{abstract}
This paper describes a prototype system to visualize and animate $3 \mathrm{D}$ scenes from car accident reports, written in French. The problem of generating such a $3 \mathrm{D}$ simulation can be divided into two subtasks: the linguistic analysis and the virtual scene generation. As a means of communication between these two modules, we first designed a template formalism to represent a written accident report. The CARSim system first processes written reports, gathers relevant information, and converts it into a formal description. Then, it creates the corresponding $3 \mathrm{D}$ scene and animates the vehicles.
\end{abstract}

\section{Introduction}

This paper describes a prototype system to visualize and animate a 3D scene from a written description. It considers the narrow class of texts describing car accident reports. Such a system could be applied within insurance companies to generate an animated scene from reports written by drivers. The research is related to the TACIT project (Pied et al., 1996) at the GREYC laboratory of the University of Caen and ISMRA.

There are few projects that consider automatic scene generation from a written text, although many projects exist that incorporate natural language interaction in virtual worlds, like Ulysse (Bersot et al., 1998; Godéreaux et al., 1999) or AnimNL (Badler et al., 1993). Visualizing a written car accident report requires a different approach. It is closer to projects focusing on text-to-scene conversion, like WordsEye (Coyne and Sproat, 2001). However, unlike the latter, our objective is to build an animation rather than a static picture and behavior of dynamic objects must then be taken into account. There also exist systems that carry out the reverse processing, from video data to text description, as ANTLIMA (Blocher and Schirra, 1995).

We present here an overview of the CARSIM system that includes a formalism to describe and represent car accidents, a linguistic module that summarizes car accident reports according to this formalism, and a visualizing module that converts formal descriptions to 3D animations. In our case, the linguistic module has to deal with texts where syntax and semantics involve time and space description and simultaneous actions of two or more actors (i.e. the cars).

The remainder of our paper is organized as follows. Section 2 presents the formalism for describing an accident. Section 3 describes the template filling methods that lead to the conversion of a text into its formal representation. Section 4 covers planning techniques and accident modelling algorithms that we use. Finally, Section 5 presents and discusses the evaluation of the system on the test corpus (MAIF corpus).

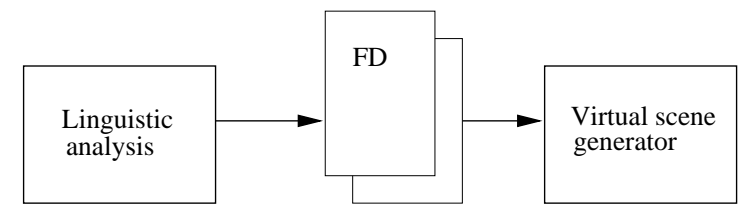

Figure 1: The two subsystems and the FD (Formal Description) as a means of communication.

\section{Formal Representation in CarSim}

"Véhicule B venant de ma gauche, je me trouve dans le carrefour, à faible vitesse environ $40 \mathrm{~km} / \mathrm{h}$, quand le véhicule $\mathrm{B}$, percute mon véhicule, et me refuse la priorité à droite. Le premier choc atteint mon aile arrière gauche, sous le choc, et à cause de la chaussée glissante, mon véhicule dérape, 
et percute la protection métallique d'un arbre, d'où un second choc frontal." Text A4, MAIF corpus.

"I was driving on a crossroads with a slow speed, approximately $40 \mathrm{~km} / \mathrm{h}$. Vehicle $\mathrm{B}$ arrived from my left, ignored the priority from the right and collided with my vehicle. On the first impact, my rear fender on the left side was hit and because of the slippery road, I lost control of my vehicle and hit the metallic protection of a tree, hence a second frontal collision." Text A4, MAIF corpus, our translation.

The text above is an accident report from the MAIF $^{1}$ corpus, which contains 87 texts in French. It is a good example of the possible contents of an accident description: a rather complex interaction between a set of different objects (two cars and a tree). This section describes the formal representation used in the CARSIM system. The example of Text A4 will be explained with more details in Section 2.5.

\subsection{The General Accident Model}

In CARSim, the general accident model consists of three lists of objects: motionless objects (STATIC), moving objects (DYNAMIC), and finally collisions (ACCIDENT).

STATIC and DYNAMIC lists describe the general environment in which the accident takes place. Knowing them, the accident itself is the only remaining item to determine. Using manual simulation, we realized that most accidents in the corpus could be framed using an ordered list of collisions $^{2}$. Each collision is represented by a relation between two objects either in DYNAMIC and/or STATIC lists

\section{$2.2 \quad$ Static Objects}

In general, a static object can be defined with two parameters: one defining the nature of the object and another one that defines its location. In CARSIM, a static object can be either a road type or an object that can participate in a collision (e.g. a tree). In the formal description, a reference to the latter kind of object can occur in a collision specification. This is why these static objects are defined with an identity parameter (ID).

Concerning ROAD objects, their nature is specified in the KIND parameter. The possible KIND values in the present prototype are: crossroads, straightroad, turn_left, and turn_right.

TREEs, LIGHTs (traffic lights), STOPSIGNs, and CROSSINGs (pedestrian crossings) are the

\footnotetext{
${ }^{1}$ Mutuelle Assurance Automobile des Instituteurs de France. MAIF is a French insurance company.

${ }^{2}$ Two collisions will never happen at the same time.
}

other possible static objects. Their location is given by the COORD parameter. Since trees and traffic lights can participate in collisions, they also have an ID, that allows further references. Finally, traffic lights contain a COLOR parameter to indicate the color of the light (red, orange, green or inactive).

\subsection{Dynamic Objects}

Dynamic objects cannot be defined by giving only their nature and position. Rather than the position, the movement of the object must be defined.

In the CARSIM formal representation, each dynamic object is represented by a VEHICLE, with a KIND parameter indicating its nature, (car or truck) and a unique identifier ID. The movement of a dynamic object is defined by two parameters. The INITIAL DIRECTION defines the direction to which the object is headed before it starts driving (north, south, east, or west). The second parameter is an ordered list of atomic movements that are described by EVENTs. This list is called the event chain and corresponds to the CHAIN parameter. KIND specifies the nature of each event. At present, CARSIM recognizes the following events: driving_forward, stop, turn_left, turn_right, change_lane_left, change_lane_right, overtake.

Figure 2 shows the motion of a dynamic object with KIND $=c a r$, INITIAL DIRECTION = East

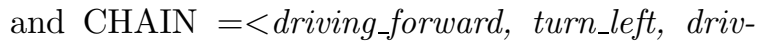
ing_forward $>$.

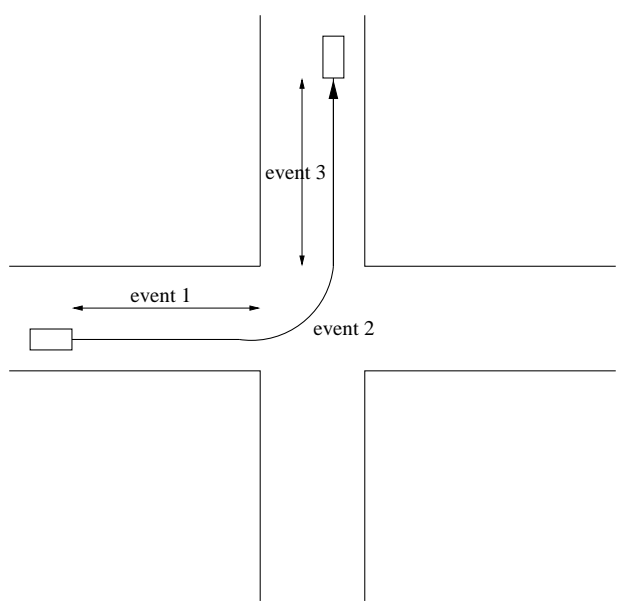

Figure 2: A crossroads with a vehicle driving forward, turning left and driving forward with an initial direction to the East.

\subsection{Collisions}

As we said before, the accident is described by an ordered list of collisions. The order of the 
collisions in the list corresponds to the order in which they take place in the accident simulation. A collision is defined by giving the two objects that participate in the collision and some additional attributes. At present, these attributes are the collision coordinates and the parts of the vehicles that are involved in the collision (participating parts).

There is a slight distinction between the vehicle that collides (in other words: the actor) and the vehicle that is hit (the victim). For planning reasons (and also for linguistic grounds) it is useful to maintain this distinction in the formalism. To summarize, a collision occurs between an actor and a victim. The victim can be either a static or a dynamic object, the actor clearly has to be a dynamic object. The notions of actor and victim are not related with the responsibility of one particular vehicle within the accident. This kind of relationships must be deduced from a complex responsibilities analysis, that could be based on the traffic rules.

Next to the location (coordinates) of the collision, something has to be said about the configuration of the objects while colliding. The participating parts are sometimes given in the text, see for example Text A4 at the beginning of this section. The CARSim system uses a simplified model of these vehicle parts. They are divided in four categories: front, rear, leftside, and rightside, plus one unknown category.

\subsection{An Example}

In order to give an example of a formal accident description and also to introduce the linguistic part, we will give now more details about the manually written FD of Text A4.

In a written text, information can be given either explicitly or implicitly. Besides, the contents of implicit information differs in each text. In Text A4, what information can we directly gather from the text?

Text A4 describes an accident with two collisions, involving two vehicles and a tree. It takes place at a crossroads. The first collision involves two vehicles. One of them is referred to as the "vehicle B", the other is the narrator's vehicle ("my vehicle"). From now on, vehicles will be called vehicleB and vehicleA respectively. The second collision involves vehicle $A$ and the tree. In the FD, the tree is identified in a unique way as tree1. From this information, we already know how many objects will be needed to describe the accident: two static objects (a crossroads and a tree tree1), two dynamic objects (vehicleB and vehicleA) and finally two collisions.
The text does not mention any special behavior of the two vehicles. They are both driving when the accident occurs. Hence, the event chain is the same for both vehicles, a single driving_forward event.

The roles played by the vehicles in each collision are also given. As human beings, we deduce them from the grammatical functions of the noun groups or pronouns referring to the vehicles in the sentences where collisions are described. In the first collision, the actor is vehicle $B$ and the victim vehicleA (respectively, subject and object of the verb "percuter", "to collide with" in the translation). In the second one, the actor is vehicle $A$ and the victim tree1.

The parts of the vehicles that participate in a collision are sometimes explicitly given in the report, as for example for vehicleA in Text A4. In the first collision, the impact occurs at the rear left-hand side of the vehicle ("On the first impact, my rear fender on the left side was hit") and in the second one, vehicleA hits the tree with the front of the car ("hence a second frontal collision").

Actually, we don't know whether the vehicles in the text are cars, trucks or something else. As no precise information is explicitly given in the text, we simply assume that these vehicles are $\operatorname{cars}^{3}$. The type of vehicles is not the only implicit piece of information in the text. The initial directions of the vehicles are only known relatively to each other. We know that vehicleB is coming from the left-hand side of vehicleA ("Vehicle B arrived from my left") and if we arbitrary decide that vehicleA starts heading to the North, then vehicleB has to start heading to the East. The same fragment of the text gives us the participating part of vehicleB. Since the participating part of vehicle $A$ in the first collision is leftside, we can conclude that vehicleB's part is front. The tree has no particular participating part. Thus, it will be defined as unknown but we can assume that the impact occurs with the trunk because all the scene takes place in a two-dimensional plane.

Below is the formal description of Text A4 that can be given to the simulation module of CARSIM:

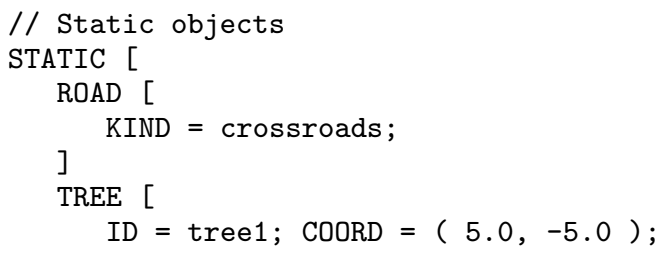

${ }^{3}$ car will be the default value of the KIND parameter of dynamic objects. 
]

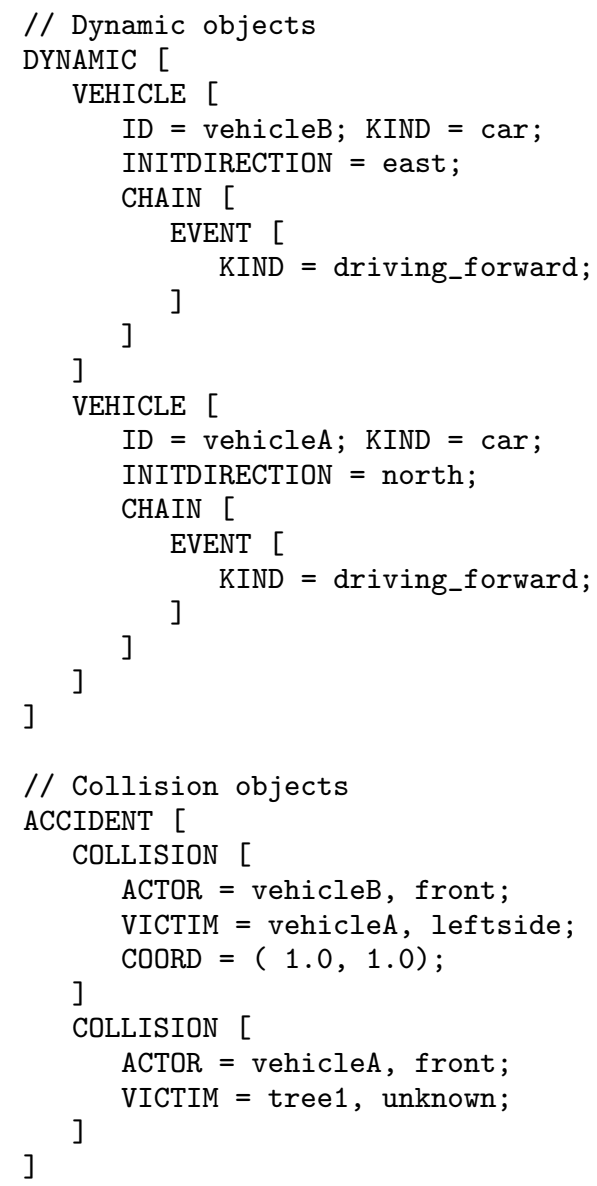

The only information we did not discuss yet are the coordinates of static objects and impacts. Coordinates are numbers. They are never explicitly given in the text and obviously, even if some numbers appeared in the text, the semantic of these numbers would be implicit too. CARSIM assumes that coordinates $(0,0)$ are the center of the scene. In Text A4, the origin is the center of the crossroads. The first collision occurs in the crossroads, hence the coordinates will be close to the origin. The coordinates of the tree are chosen so that they match the idea of the scene as a reader could imagine it. They also depend on the size of the graphical objects that are used in the 3D scene (e.g. the size of the roads).

\section{The Information Extraction Task}

The first stage of the CARSIM processing chain is an information extraction (IE) task that consists in filling a template corresponding to the formal accident description (FD) described in Section 2. Such systems have been already implemented, as FASTUS (Hobbs et al., 1996), and proved their robustness. Our information retrieval subsystem is restricted to car accident reports and is goaldriven. The main idea is to start from a default description, a pre-formatted FD, that the IE task alters or refines using inference rules. Hence, the default output will be a well-formatted FD, describing a collision between two cars, even if the given text is a poem.

\subsection{Parsing}

The first step of the information extraction process is a lexical analysis and a partial parsing. The parser generates tokenized sentences, where noun groups, verb groups, and prepositional groups are extracted. The parser uses DCG rules (Pereira and Shieber, 1987) and a dictionary containing all the words that occur in the corpus.

\subsection{Extracting Static Objects}

The formalism describes two types of static objects: the type of road (the road configuration) and some other static objects (stop signs, traffic lights, pedestrian crossings and trees). The method used to extract these objects consists in looking up for keywords in the tokenized text.

The extraction of static objects is done at the beginning of the information extraction task. We realized that the road configuration is the most relevant piece of information in the description of an accident, since it conditions all the following steps (see Section 3.4 for further explanations).

The formalism considers four different configurations: straightroad, crossroads, turn_left, and turn_right. In the present system, we restricted it to three types of road:

- crossroads, indicated by cue words such as "carrefour", "intersection", "croisement" (crossroads, intersection, junction).

- turn_left, with cues such as "virage", "courbe", "tournant" (bend, curb, turn). We assume that turn_left and turn_right are equivalent.

- straightroad, that corresponds to the situation when none of the previous words have been found.

\subsection{Extracting Collisions}

A collision consists of a verb, an actor, a victim and of the participating parts of the two vehicles. We select verbs describing a collision such as "heurter" ("to hit"), "taper" ("to bang"), "percuter" ("to crash into"), "toucher" ("to touch"),...

For each extracted verb, the system checks whether the verb group is in passive or active 
form, then identify the related grammatical relations: subject-verb and verb-object or verb-agent. Extraction techniques of such dependencies have already been implemented, as in (Aït-Mokhtar and Chanod, 1997). Our system uses three predicates in order to find the subject (find_subject) and either the object (find_object) or the agent (find_agent) of the verb. If the verb is in an active form, it makes the assumption that the subject and the object of the verb will be respectively the actor and the victim of the collision. In the case of a passive form, the subject will be the victim and the agent, the actor.

Below is the sketch of the algorithm of these three predicates:

- find_subject looks for the last noun group before the verb that describes a valid actor, that is a vehicle or a personal pronoun like "je" ("I"), "il" ("he"), or "nous" ("we") .

- find_object starts looking for the first noun group after the verb that describes a valid victim, that is both vehicles and static objects. If no valid victim is found, it searches for a reflexive or personal pronoun inside the verb group. In case of failure, the first noun group after the verb is chosen.

- find_agent looks for a valid actor in a prepositional group introduced by "par" ("by").

\subsection{Generating Collisions and Dynamic Objects}

For each collision, the system tries to extract the participating parts of the vehicles in the noun groups that refer to the actor and the victim. To do this, it looks for cues like "avant", "arrière", "droite", or "gauche" ("front", "rear", "right", or "left").

Then, the system creates two dynamic objects (see Section 3.5) and a collision between them. The generated properties of the collision depend on the road configuration:

- Straight road: the first vehicle heads to the East, the other one starts from the opposite end of the road, heading to the West. The collision is a head-on impact.

- Turn: The first vehicle starts heading to the East, then turns to the Left. The second one starts heading to the South, then turns to the Right. The collision is frontal and happens at the center of the turn.

- Crossroads: We choose to represent here the most frequent traffic offence (in France). The first vehicle drives straight to the East, the second one drives to the North. The front of the actor's vehicle collides with the left-hand side of the victim.

As we do not extract the initial directions of the vehicles, these three cases are the only possible ones. When the system cannot find the actor or the victim of a collision, default objects are created matching the road configuration.

\subsection{Deleting Useless Objects}

When creating collision objects, two new vehicles are instantiated for each collision, even if the victim is a static object. Moreover, one vehicle can obviously participate in several collisions. All the unnecessary vehicles should then be thrown away.

A vehicle that represents a static object can be removed easily, since the real static object still exists. All we have to do is to modify the reference given in the victim parameter of the collision in the template, then delete the redundant vehicle.

Deleting the duplicates is more difficult and involves a coreference resolution. An identification mechanism of the narrator has been added to the system. All the personal pronouns in the first person or some expressions like "the vehicle A" will be designated with the id enunciator. In the other cases, coreference occurs only when the two ids are strictly the same (in the sense of string comparison). Then, the system keeps only the first created object between the duplicates and delete the others.

\subsection{Extracting Event Chains}

The vehicles generally do not drive straight forward. They carry out two or more successive actions. In the formal description, these possible actions correspond to the events of dynamic objects and are in limited number: driving_forward, turn_left, turn_right, change_lane_right, change_lane_left, overtake, and stop.

In written reports, these actions are mostly indicated by verbs. The system has to identify them and to link the corresponding event(s) to the appropriate vehicle. When the subject is identified as the narrator, the link is obvious. In the other cases, if there are only two vehicles, the narrator and another one, a new event is added to the event chain of the second vehicle. Otherwise, the system checks whether the subject of the verb is strictly identical (string comparison) to one vehicle's id. In this case, a new event is also created and added to the event chain. Some verbs imply multiple events, e.g. "redémarrer" ("to get driv- 
ing again") that indicates that the driver stopped beforehand. Consequently, a stop event then a driving_forward event are added.

With this simple extraction mechanism, the order of the events in the event chain does not necessarily respect the chronology but rather the order of the text. We assume that the story is linear, which is the case in most accident reports.

\subsection{Writing the Formal Description}

The final step of the linguistic part consists in formatting a template corresponding to the accident description. Because the inferred facts have exactly the same attributes as the formalism's elements, a very simple transcription algorithm is used to convert the facts in a text file that can be processed afterwards by the simulator.

\section{Planning}

Planning complex events like collisions requires a well-defined and flexible planning architecture. General planning algorithms which apply methods incorporating artificial intelligence, are discussed in (Nilsson, 1998). The CARSIM planner is much more straightforward, because the planning process is not as complex as a lot of traditional AI planning problems, see also (Norvig and Russell, 1995). The total planning process is performed by using five different subplanners, which all perform a small part of the total planning task.

\subsection{The Preplanner}

The preplanner is a planner that ensures the consistency of the formal description. If some values are not given (e.g. coordinates of a static object or initial directions of dynamic objects) or some values imply a contradiction (a vehicle turning left on a straight road), this planner tries to find (default) values and to solve the conflicts. This planner is a simple knowledge base, as discussed in (Norvig and Russell, 1995).

\subsection{The Position Planner}

The position planner estimates the start and end positions of the vehicles in the simulation. By default, a vehicle is placed 20 meters away from the center of the (cross)road. If two or more vehicles are moving in the same direction, they can't all be placed at this distance because they would overlap. Therefore, if there is more than one vehicle facing a particular direction, the second vehicle is placed at a distance of 26 meters from the center and if there is a third vehicle, it is placed at 32 me- ters from the center ${ }^{4}$. Regarding the end points of the vehicles, the vehicle that is placed closest to the center, will have its end point placed farther away from the center. The vehicle initially having a start point far away from the center will have an end point close to the center, so that every vehicle traverses approximately the same distance.

\subsection{The Trajectory Planner}

Based on the (very global) description of the movement of every vehicle in the formal model, this planner constructs a trajectory, represented by a set of points in the Euclidian space. Every event in the event chain is converted to a list of trajectory points. A turn is approximated by a number of points lying on a circle arc. Overtaking is modelled by using a goniometrical function.

\subsection{The Accident Planner}

The accident planner uses the trajectory that is created by the trajectory planner. Since event chains only include atomic movements and not collisions, this trajectory is planned as if there was no collision at all. The task of the accident planner is to change this trajectory in such a way that it incorporates the collision. Some part of it has to be thrown away and an alternative part (which ultimately leads to the point of collision) has to be added to the trajectory. For every vehicle, actor or victim, the trajectory is thus changed in two steps:

1. Remove a part of the trajectory.

2. Add a part to the trajectory so that the final result will be a trajectory that leads the vehicle to the point of collision.

The part of the trajectory that has to be removed depends on the coordinates where the collision occurs. We designed an algorithm that draws a circle around the collision point and removes the trajectory part that lies within the circle region. Also, the segment that comes after the removed trajectory part is thrown away, because a trajectory does not allow gaps. The radius of the circle is thus a parameter that defines the precision of the algorithm. If a large radius is chosen, a large part of the trajectory will be removed. An application of the algorithm using a small radius only removes the trajectory part closest to the collision point.

\footnotetext{
${ }^{4}$ In the CARSIM system, the maximum number of vehicles that can have the same initial direction is three.
} 


\subsection{The Temporal Planner}

The temporal planner of the CARSIM system is not a planner in the sense of the planners described in (Nilsson, 1998) The temporal planner of the CARSim system plans the temporal values of the trajectory in two steps. Generally, a trajectory consists of a number of 'normal' trajectory points, followed by a number of trajectory points that represent a collision. First the segment that is not part of any collision is planned. After that, the system plans the remaining segment. In the CARSim system, every trajectory point has a time value. This is a value between 0 and 1 , with 0 representing the beginning of the simulation and 1 being the end of it. The temporal planner tries to find time values for the trajectory points so that the collisions happen in a natural way.

\section{Results and Discussion}

The CARSim system has been implemented and evaluated over the MAIF corpus. The assessment method does not consist, as usually done with IE systems, in calculating a precision and a recall. Our objective is to design a system that carries out the whole processing chain, that is from a written report up to a $3 \mathrm{D}$ animation. Therefore, we preferred to compare the simulation with the understanding and mental representation of the scene that could have a human reader. This implies that some aspects of the formal description are not taken into account when evaluating the system, e.g. we assume that the value of the INITIAL_DIRECTION parameter is less important than the positions of the vehicles relatively to each other. Hence, we considered that the result is acceptable as far as the latter is correct.

According to such criteria, we considered that the simulation provided by the system corresponds, in $17 \%$ of the texts, with what could have imagined a human being. Figure $3 \& 4$ show the two collisions described in Text A4.

Failure cases have many different grounds. They may be related either to the IE task, to the simulator, or to a lack of cooperation between the two subsystems. Evaluating separately each subsystem leads to a better understanding of the actual limits of the system.

Feeding the simulator with manually written formal descriptions provides a good way to evaluate it for itself. According to such tests, the CARSIM system generates an acceptable simulation of almost $60 \%$ of the reports. This implies that the results of the overall system will be lower. CARSim's simulator does not succeed in simulating manually written formal descriptions because

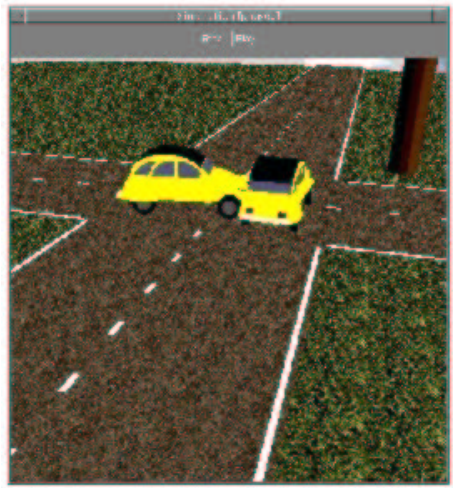

Figure 3: The first collision in Text A4.

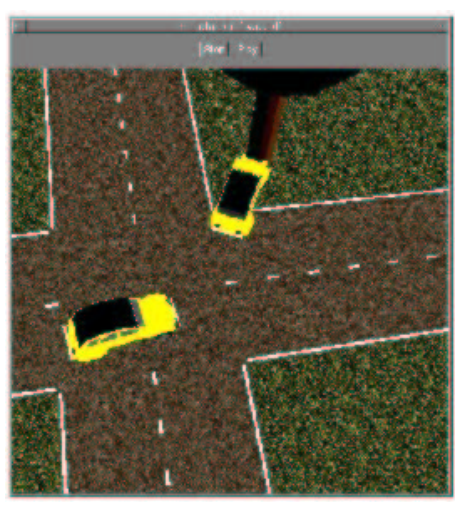

Figure 4: The second collision in Text A4.

of three main causes: expressivity of the formalism that does not cover all possible accidents (e.g. synchronization between event chains of different objects), the restricted number of scenarios considered by the CARSIM visualizer and the limited database of 3D graphical objects. Depending on the text, the failure is the result of either only one of these restrictions or a combination. Future work on the project will focus on these issues.

The efficiency of the IE task varies with the nature of extracted information. First, the results clearly depend on the accuracy with which the system can correctly extract impacts, that is find the verb representing the collision and also resolve the actor, the victim and possibly their participating parts ${ }^{5}$. This task is successfully accomplished in $69 \%$ of the texts ${ }^{6}$. In addition, the system correctly extracts EVENTS in $35 \%$ of the texts. This means that in $35 \%$ of the texts, all the events are properly extracted with a good ordering.

\footnotetext{
${ }^{5}$ when the parts are explicitly described

${ }^{6}$ In the rest, it generates default impacts or impacts are erroneous.
} 
Concerning time and space information, the system provides only simple mechanisms to obtain them. Our system is at an early stage and our objective when designing it was to see whether such an approach was feasible. It represents a sort of improved baseline with which we can compare further results. At this time, the temporal information known by the system is restricted to the events associated with dynamic objects. Our method assumes that they are given in the text in the same order they occur in reality. This is a simplification that proves wrong in some reports. Further improvements could take into account tenses of verbs, temporal adverbs and prepositions, so that the system could determine the real chronological relationships between events.

A similar comment can be given with regards to spatial information. In CARSIM, the spatial configuration (the background of the scene) is given mainly by the type of roads. The extraction of participating parts also provides additional information that influence the relative positions of the vehicles when colliding. During preplanning stage, the system checks the consistency of the FD and tries to resolve conflicts between the different information. At present, initial directions of the vehicles depend only on the background of the scene, that is the road configuration. The coordinates are also chosen arbitrary from the beginning. See for example the tree referred as tree1 in Text A4: no information about its location is given in the text. The only facts relative to it that we can deduce from the original report are its existence and its involvement in a collision. Moreover, the problem of choosing a referential from which to calculate coordinates is quite unsolvable for texts that do not mention it explicitly. The IE task could involve deeper semantic analysis that provides means of constructing a more global spatial representation of the scene.

\section{Conclusion}

This paper has presented a prototype system that is able to process correctly $17 \%$ of our corpus of car accident reports up to a $3 \mathrm{D}$ simulation of the scene. The chosen approach divides the task between information extraction to fill templates and planning to animate the scene. It leads to encouraging results, considering that the information retrieval could be improved by integrating more elaborate methods to deal with space and time in written texts.

\section{References}

S. Aït-Mokhtar and J-P. Chanod. 1997. Subject and object dependency extraction using finitestate transducers. In Proceedings of $A C L$ workshop on Automatic Information Extraction and Building of Lexical Semantic Resources for NLP Applications.

N. Badler, W. Becket, B. Di Eugenio, C. Geib, L. Levison, M. Moore, B. Webber, M. White, and X. Zhao. 1993. Intentions and expectations in animating instructions: the AnimNL project. In Intentions in Animation and Action. Institute for Research in Cognitive Science, University of Pennsylvania, March.

O. Bersot, P.O. El-Guedj, C. Godéreaux, and P. Nugues. 1998. A conversational agent to help navigation and collaboration in virtual worlds. Virtual Reality, 3(1):71-82.

A. Blocher and J.R.J. Schirra. 1995. Optional deep case filling and focus control with mental images: ANTLIMA-KOREF. In Proceedings of IJCAI-95, pages 417-423.

R.E. Coyne and R. Sproat. 2001. Wordseye: An automatic text-to-scene conversion system. In Proceedings of International Conference on Computer Graphics and Interactive Technologies (SIGGRAPH 2001). AT\&T Research Lab.

C. Godéreaux, P.O. El-Guedj, F. Revolta, and P. Nugues. 1999. Ulysse: An interactive, spoken dialogue interface to navigate in virtual worlds, lexical, syntactic, and semantic issues. In John Vince and Ray Earnshaw, editors, Virtual Worlds on the Internet, chapter 4, pages 53-70. IEEE Computer Society Press.

J.R. Hobbs, D. Appelt, J. Bear, D. Israel, M. Kameyama, M. Stickel, and M. Tyson. 1996. FASTUS: A cascaded finite-state transducer for extracting information from natural-language text. In Roche and Schabes, editors, Finite State Devices for Natural Language Processing. MIT Press.

N.J. Nilsson. 1998. Artificial Intelligence, a New Synthesis. Morgan Kaufmann Publishers, Inc.

P. Norvig and S.J. Russell. 1995. Artificial intelligence: a modern approach. Prentice Hall.

F.C.N. Pereira and S.M. Shieber. 1987. Prolog and Natural Language Analysis. Stanford University. CSLI Lecture Notes No.10.

F. Pied, C. Poirier, P. Enjalbert, and B. Victorri. 1996. From language to model. In Workshop Corpus-Oriented Semantic Analysis in European Conference on Artificial Intelligence (ECAI), August. 